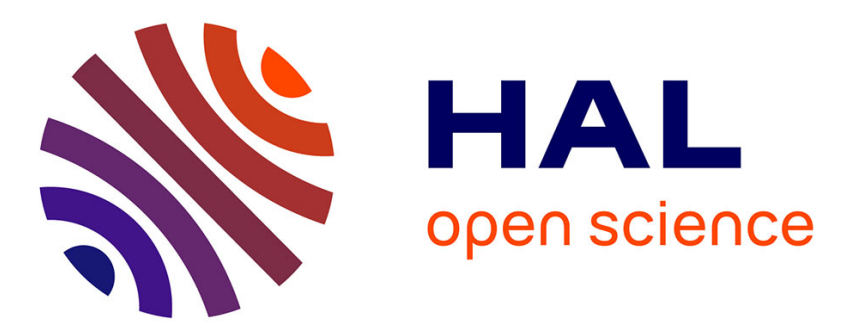

\title{
Sensitive colored hybrid inorganic/organic pigments based on polymer-coated microsized particles
}

\author{
L. Ghannam, H. Garay, Laurent Billon
}

\section{To cite this version:}

L. Ghannam, H. Garay, Laurent Billon. Sensitive colored hybrid inorganic/organic pigments based on polymer-coated microsized particles. Macromolecules, 2008, 41 (20), pp.7374-7382. 10.1021/ma800522k . hal-01582036

\section{HAL Id: hal-01582036 https://hal.science/hal-01582036}

Submitted on 1 Jun 2021

HAL is a multi-disciplinary open access archive for the deposit and dissemination of scientific research documents, whether they are published or not. The documents may come from teaching and research institutions in France or abroad, or from public or private research centers.
L'archive ouverte pluridisciplinaire HAL, est destinée au dépôt et à la diffusion de documents scientifiques de niveau recherche, publiés ou non, émanant des établissements d'enseignement et de recherche français ou étrangers, des laboratoires publics ou privés. 


\title{
Sensitive Colored Hybrid Inorganic/Organic Pigments Based on Polymer-Coated Microsized Particles
}

\author{
Léila Ghannam, ${ }^{\dagger, \$, \S}$ Hélène Garay, ${ }^{\ddagger}$ and Laurent Billon*,† \\ Institut Pluridisciplinaire de Recherche sur l'Environnement et les Matériaux, Equipe de \\ Physico-Chimie des Polymères, IPREM/EPCP UMR 5254, Hélioparc, 2 avenue Angot, \\ 64000 Pau, France, Centre de Matériaux de Grande Diffusion, Ecole de Mines d'Alès, \\ Hélioparc, 2 avenue Angot, 64000 Pau, France, and Département de Chimie, et Biochimie, \\ Faculté des Sciences, Université Libanaise, Beyrouth, Lebanon
}

\begin{abstract}
We present herein the preparation of colored hybrid organic/inorganic pigments: poly(styreneco-alizarin)/mica. We have used direct nitroxide-mediated radical polymerization of styrene and modified alizarin from mica-bound bicationic azo compounds as initiator. The color of the $\mathrm{P}(\mathrm{S}-\mathrm{co}-\mathrm{A})-$ mica composites is related both to the adsorption density of polymers on mica surface and to the length of the macromolecular chains. The longer the polymerization time is, the more the chains are crushed onto the mica surface and the nearer the dye is to the surface. Consequently, the dye will be strongly influenced by the oxanions of the surface leading to color variation. This variation has been attributed to the superimposition of different adsorption of the alizarin due to different states: when closer to the surface, alizarin is more influenced by oxanions and its color tends to pink, when farther away from the surface, the alizarin electronic state is less changed and its color tends to yellow. Finally, the global color is due to the mix of the different electronic states leading to orange pigments. This behavior could be represented as a "gradient" of alizarin position from the mica surface to the air/polymer interfaces. Depending on the synthesis methodology, the color of such pigments can be easily tuned not only by adjustment of the length and the grafting density but also by the number of dyes in the chains.
\end{abstract}

\section{Introduction}

Polymerization with surface-bound initiators is a potentially flexible and convenient method for modifying the surface properties of inorganic materials, improving interfacial bonding in composites, and preparing novel inorganic-organic hybrids. $^{1,2}$

The development of such materials aimed in general to improve the properties of polymers such as the following: mechanical properties, ${ }^{3}$ thermal stability ${ }^{4,5}$ and flame behavior $^{6-8}$ with or without interfoliation of silicate by polymers.

Recently, the surface initiated classical or controlled free radical polymerization techniques of polymer from inorganic nanoparticle surfaces have been established. Notably, Suter and co-workers have described the graft polymerization of styrene on mica surface..$^{6-8}$ Von Werne and Patten have also reported the atom transfer radical polymerization of styrene from the surface of colloidal silica particles. ${ }^{9,10}$ Polystyrene-grafted magnetite nanoparticles prepared through surface-initiated nitroxide-mediated radical polymerization have been demonstrated by Takahara. ${ }^{11,12}$ More recently, Armes et al. have described a robust polymer brushes approach from a planar mica surface by ATRP. A cationic macroinitiator strongly adsorbed onto the mica was used to reinitiate the polymerization of a biomimetic zwitterionic monomer. ${ }^{13}$

Among the possible techniques that allow the synthesis of well-defined grafted homopolymers and copolymers by a controlled free-radical polymerization (CRP) process, ${ }^{14}$ nitroxide mediated polymerization is one of the efficient methods to elaborate hybrid inorganic/organic materials. ${ }^{15-19}$

\footnotetext{
* Corresponding author. E-mail: laurent.billon@univ-pau.fr.

†nstitut Pluridisciplinaire de Recherche sur l'Environnement et les Matériaux, Equipe de Physico-Chimie des Polymères, IPREM/EPCP UMR 5254 .

† Centre de Matériaux de Grande Diffusion, Ecole de Mines d'Alès.

§ Département de Chimie, et Biochimie, Faculté des Sciences, Université Libanaise.
}

In a previous paper, we have already demonstrated the possibility of synthesizing poly((butyl acrylate)- $b$-(styrene- $c o$ alizarin)) from mica particles ${ }^{20}$ using a modified surface with poly(n-butyl acrylate) adsorbed from the mica surface, as macroinitiator. ${ }^{21}$ With that method, it was possible to generate some adsorbed diblock copolymer from the mica surface under CRP, controlling the length of the first adsorbed polymer onto the mica particle. Furthermore, a pink-colored composite was created by adsorbing a poly (BA- $b-(\mathrm{S}-c o-\mathrm{A}))$ diblock. ${ }^{21}$ Since the color of the free poly(BA- $b$-(S-co-A)) diblock (not adsorbed on mica) was yellow, we supposed that the pink color of the composite obtained could be due on one hand to the $\mathrm{pH}$ dependence of the dye (modified alizarin) and on the other hand to the effect of oxanions of the mica surface.

The general theme of the research reported here concerns the following points:

1. The preparation of colored hybrid poly(styrene-coalizarin)-mica materials by direct nitroxide-mediated radical polymerization of styrene and modified alizarin from micabound bicationic azo compound as initiator.

2. The relationship between the adsorption density of polymer on mica surface and the resulting color of the $\mathrm{P}(\mathrm{S}-\mathrm{Co}-\mathrm{A})-$ mica composites.

This paper describes, for the first time, the synthesis of colored $\mathrm{P}(\mathrm{S}-\mathrm{co}-\mathrm{A})-$ mica composite pigments and the correlation of

Scheme 1. Chemical Structures of the Alizarin-Based Monomers Keeping the Hydroxyl Function in Ortho and Meta Positions, Respectively Left to Right

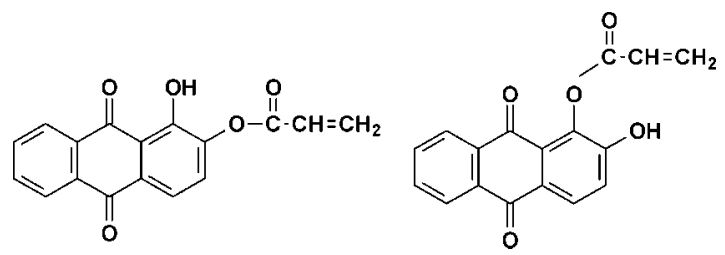



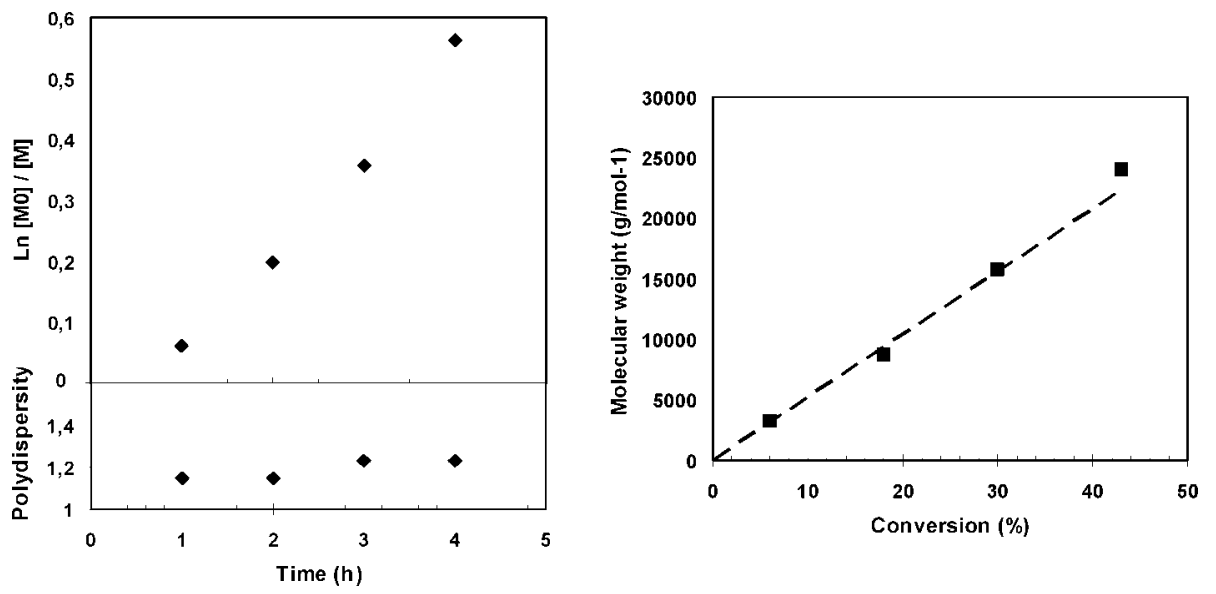

Figure 1. (a) Evolution of $\ln \left([\mathrm{M}]_{0} /[\mathrm{M}]\right)$ and $I_{\mathrm{p}}$ vs time and (b) evolution of $M_{\mathrm{n}}$ vs conversion of the copolymerization of $\mathrm{S}$ and A carried out at 120 ${ }^{\circ} \mathrm{C}$ in the presence of SG1.

adsorption density and macromolecular length of polymers on the color of these new pigments.

\section{Experimental Section}

Materials. The soft mica samples were received from the "Comptoir des Minéraux et des Matières Premières" CMMP (France). The physicochemical characteristics of this mica were given in a previous study. ${ }^{20,21}$ The main characteristics are a density value of $2.80 \mathrm{~g} / \mathrm{cm}^{3}$, a $\mathrm{pH}$ value around 9 when dispersed in water (characteristic of a basic surface strongly charged with anionic $\left.\mathrm{SiO}^{-} \mathrm{X}^{+}\left(8 \% \mathrm{Na}^{+}, 92 \% \mathrm{~K}^{+}\right)\right)$, an average particle size of $45-150$ $\mu \mathrm{m}$ and a low specific surface value of $5.9 \mathrm{~m}^{2} / \mathrm{g}$.

The initiator used was a water-soluble bicationic azo compound previously reported for the preparation of polymer-clay composites, 2, 2'-azobis (iso-butyramidine hydrochloride) (AIBA) (DupontUSA). ${ }^{6-8,20-22}$

The counter-radical $\mathrm{SG}_{1}(90 \%)$ was received from ArkemaFrance. The monomer and solvents mentioned in the present paper were used as received from Aldrich.

The dye chosen for this study is the modified "alizarin" (A) which is an anthraquinone. The chemical modification and the characterization of this dye have been described in a previous paper. ${ }^{20} \mathrm{We}$ have shown the presence of two modified structure types of alizarin after modification. The first one is the alizarin keeping the $\mathrm{OH}$ function in ortho position (35\%) and the second one in meta position $(65 \%)$. The chemical structures of the modified alizarin A used in this study as monomers are represented in Scheme 1.

Here, it is important to notice the presence in these two structures of acid groups due to the presence of the residual phenol functions (hydroxyl group on aromatic ring). The $\mathrm{p} K_{\mathrm{a}}$ value of these molecules is around 9 which corresponds to a strong acid able to form an alcoxy group $\mathrm{O}^{-}$as conjugated base.

Polymerization on Mica Surface. In order to obtain poly(styreneco-alizarin) "P(S-co-A)" at the surface of mica, two steps are necessary: (i) modification of mica surface by adsorption of a cationic initiator; (ii) copolymerization of styrene and modified alizarin (A) from the initiator-bound mica. ${ }^{6-8}$

For this purpose, $500 \mathrm{mg}$ of mica powder was dispersed in 20 $\mathrm{mL}$ of ethanol solutions of the initiator $(250 \mathrm{mg})$ at ambient temperature. The resulting suspensions were magnetically stirred overnight. After filtration, the solids were washed with $20 \mathrm{~mL}$ of ethanol, filtered again, and dried by lyophilisation for $24 \mathrm{~h}$. This hybrid material was characterized by FTIR and ${ }^{13} \mathrm{C}$ NMR CP/MAS. The amount of adsorbed initiator was determined by Thermal Gravimetric Analysis (TGA) and calculated to $1.5 \mu \mathrm{mol} / \mathrm{mg}$ of mica to adjust the $[\mathrm{S}] / 2$ [AIBA] ratio. $^{21}$

Then, a mixture of AIBA-adsorbed mica particles (166 mg corresponding to $47.4 \mathrm{mg}$ or $1.75 \times 10^{-4} \mathrm{~mol}$ of AIBA), $18.7 \mathrm{~g}$ $(0.175 \mathrm{~mol})$ of styrene $([\mathrm{S}] / 2[\mathrm{AIBA}]=500), 0.0245 \mathrm{~g}(8.33 \times$ $\left.10^{-5} \mathrm{~mol}\right)$ of SG1 (slight excess $([\mathrm{SG} 1] /[\mathrm{AIBA}]=2.1)$ ) and 0.154 $\mathrm{g}\left(5.25 \times 10^{-4} \mathrm{~mol}\right)$ of modified dye $([\mathrm{S}] /[\mathrm{A}]=333)$ was heated at $120{ }^{\circ} \mathrm{C}$ during 1,2 , and $4 \mathrm{~h}$.

After reaction, the dispersion was poured into ethanol for precipitation. After filtration and drying, the polymer-mica mixture was extracted five times with $20 \mathrm{~mL}$ of toluene to remove the nonadsorbed polymers, residual styrene $\mathrm{S}$ and alizarine A monomers. Two types of polymers were found: polymers adsorb to the mica (not removed from the mica either during polymerization or by toluene extraction) and free polymers precipitated in ethanol due to the polymer desorption as described previously. ${ }^{21}$ The solid phase was dried at room temperature under dynamic vacuum.

Characterization Methods. ${ }^{1} \mathrm{H} N M R$ spectra were recorded at $400 \mathrm{MHz}$ on a Bruker Advanced AM400 spectrometer in $\mathrm{CDCl}_{3}$ and the chemical shifts $(\delta)$ in ppm were referred to internal trimethylsilane (TMS).

Solid $U V-$ vis diffuse reflectance was recorded using a Cary UV-vis-NIR spectrophotometer (Sphere method) with a Teflon standard.

Thermal gravimetric analysis (TGA) was carried out using a System TA 2950 apparatus, to determine the amount of bound polymer chains adsorbed to mica surfaces in a temperature range $50-600{ }^{\circ} \mathrm{C}$ at a scan rate of $10{ }^{\circ} \mathrm{C} / \mathrm{min}$ in air.

The molecular weights and the polydispersity of polymers were determined by size exclusion chromatography (SEC), using a 2690 Waters System with THF as the mobile phase. Molecular weights were calculated relative to polystyrene standards.

The mica-polymer surfaces were characterized by scanning electron microscopy (SEM, Oxford Instruments, INCA ENERGY $350)$.

\section{Results and Discussion}

Free Copolymer. Macromolecular Dimensions. At first, the number average molecular weights $\left(M_{\mathrm{n}}\right)$ as well as the molar mass distribution $\left(I_{\mathrm{p}}=M_{\mathrm{w}} / M_{\mathrm{n}}\right.$, where $M_{\mathrm{w}}$ is the weight average molecular weight) of the free copolymer desorbed from the mica surface during reaction were measured by SEC. Indeed, a desorption phenomenon was previously related with polymerization time due to an increase of the affinity of the polymer chains with the reactive medium. This behavior was associated with a decrease of the ionomer character when chain length grows from the ionic initiator end chain. ${ }^{20-22}$

The kinetics of polymerization of styrene $S$ in presence of a low amount of alizarin A was achieved in order to demonstrate the ability of a bimolecular initiator system AIBA/SG1 to control the polymerization from mica surface (Figure 1). Indeed, the linearity observed in the plots of the logarithmic change of the monomer concentration with time indicates not only a first order with respect to the monomer but that the growing radical 


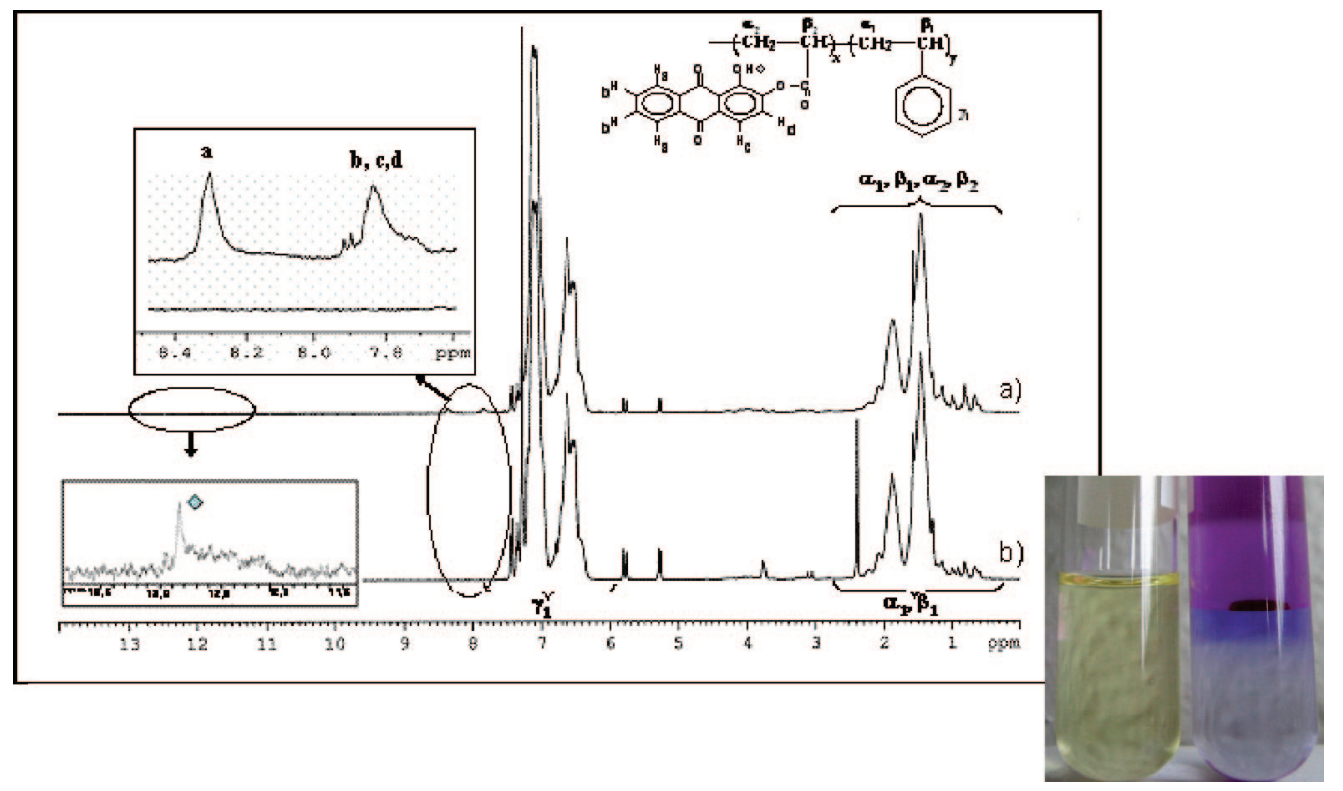

Figure 2. ${ }^{1} \mathrm{H}$ NMR spectrum of free $\mathrm{P}(\mathrm{S}-\mathrm{co}-\mathrm{A})$ (inset: test of free alizarin traces).

Scheme 2. Effect of the Alizarin Electronic Interaction with the Mica Surface

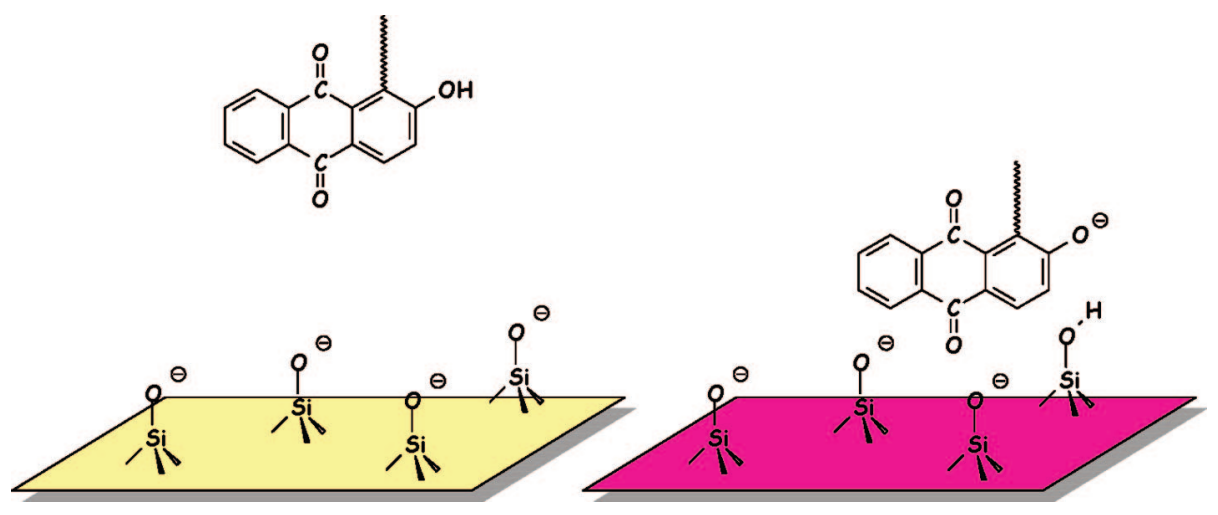

concentration remained constant (slope of the straight lines are equal to $K_{\mathrm{p}}\left[\mathrm{M}^{*}\right]$, the product of the polymerization constant $K_{\mathrm{p}}$ of these comonomers by the number of active centers $\left.\left[\mathrm{M}^{\circ}\right]\right)$, which was also ratified by the linear dependence of $M_{\mathrm{n}}$ with conversion corresponding to a constant number of living chains.

These results show that the AIBA initiator has successfully initiated the radical copolymerization of styrene $\mathrm{S}$ and modified alizarin A from the mica surfaces with a controlled behavior characterized by low polydispersity $\left(I_{\mathrm{p}}<1.2\right)$ and narrow peak shape. The chromatograms of the three free $\mathrm{P}(\mathrm{S}-\mathrm{co}-\mathrm{A})$ polymers obtained after 1,2 and $4 \mathrm{~h}$ of polymerization are reported in the Supporting Information section and the obtained $M_{\mathrm{n}}$ values are $3200\left(I_{\mathrm{p}}=1.15\right), 8700(1.15)$ and $24000 \mathrm{~g} \cdot \mathrm{mol}^{-1}(1.23)$, respectively.

Copolymers Composition. First, we can mentioned that the ethanol solution is colored after the first precipitation and that we never observed colored toluene solution after the free polymer extraction procedure. It means that the nonreacted or unlinked alizarine is extracted from the first step of the purification of the polymer-coated microsized particles. Moreover, it clearly showed the presence of residual dye for monomers conversion lower than $100 \%$, this behavior could be associated to similar reactivity ratio values of the monomer couple S/A. But in the present study, the main topic was to synthesize colored polymer electrostatically adsorbed on the mica surface with a very slight amount of dye $([\mathrm{S}] /[\mathrm{A}]=333$ corresponding to about $0.3 \%$ of A comonomer).
The ${ }^{1} \mathrm{H}$ NMR spectra of the three free polymers in $\mathrm{CDCl}_{3}$ show that the polymer structure is demonstrated by the signal at 6-8 ppm, which corresponds to the phenyl groups of PS. We present in Figure 2 an example of NMR spectrum of P(Sco-A)-2h compared to the spectrum of PS-2h synthesized in the same conditions but without modified alizarin. This ${ }^{1} \mathrm{H}$ NMR spectrum shows the appearance of signal characteristics of the dye monomer, signifying the incorporation of the dye into polymer chains: $\delta_{\mathrm{Ha}}=8.3, \delta_{\mathrm{Hb}, \mathrm{c}, \mathrm{d}}=7.7-7.9 \mathrm{ppm}$. Moreover, it is important to notice the presence of the signal at $\delta=12.4$ ppm characteristic of the hydroxyl function of alizarin in the ortho position. This shows that the modified dye under this structure is incorporated into the macromolecular chains of PS. The other modified alizarin structure ( $\mathrm{OH}$ in the meta position) seems to be not present. This can be explained by the steric hindrance which prevents the dye under the meta structure from polymerizing. ${ }^{20}$

In addition to this ${ }^{1} \mathrm{H}$ NMR characterization, two simple tests were made to confirm the incorporation of modified dye into polymer chains after successive washing with toluene:

1. $\mathrm{P}(\mathrm{S}-\mathrm{co}-\mathrm{A})$ was dissolved in toluene and precipitated again in ethanol. The characterization of supernatant by UV-visible spectroscopy does not show the presence of any trace of the dye, which is soluble in ethanol. It means that all the dyes present in the $\mathrm{P}(\mathrm{S}-\mathrm{co}-\mathrm{A})$ are covalently linked to the polymer chains; 


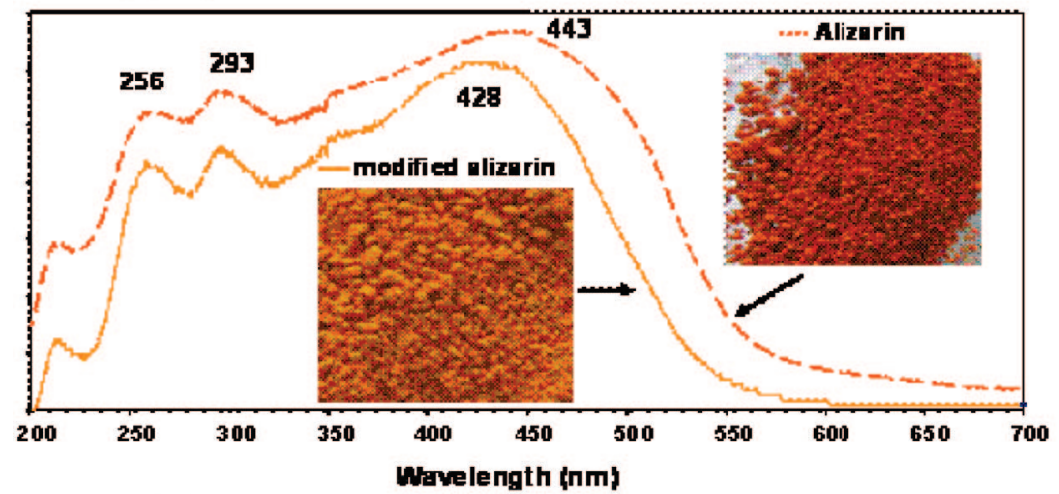

Inlensily (a.u)

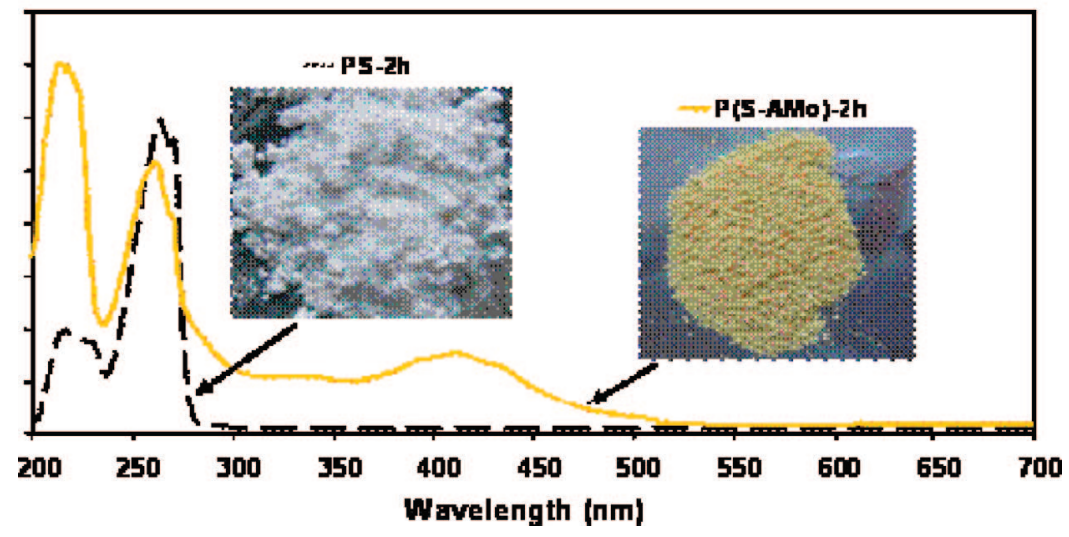

Figure 3. Photographs and UV-vis diffuse reflectance of (top) pure alizarin and modified alizarin and (bottom) pure PS and P(S-co-A).

2. In the inset of Figure 2, Alizarin being a water soluble dye, some drops of $\mathrm{NaOH}(1 \mathrm{M})$ were added to $\mathrm{P}(\mathrm{S}-\mathrm{co}-\mathrm{A}) /$ THF solution (left) until obtaining a phase separation (right). Indeed, THF and water are nonmiscible, and phase separation occurs, leading to two distinct phases. Upper purple colored organic polymer phase (top right, THF, $d=0.89$ ) and lower none colored phase (below right, water, $d=1$ ) were observed. This phenomenon means that no trace of the free dye is present in the aqueous phase; all the dye molecules are strongly linked to the macromolecular chains and then solubilized in THF. Such phenomenon is confirmed by no adsorption of the liquid aqueous phase by UV-visible spectroscopy. Moreover, it is quite clear that if the $\mathrm{P}(\mathrm{S}-\mathrm{co}-\mathrm{A})$ is yellow colored in the THF phase (left inset Figure 2), this color can be easily tuned to purple by addition of a base corresponding to the modification of the electronic state of the grafted alizarin (right inset Figure 2). This color changing alizarin ability will be discussed later in this manuscript (Scheme 2).

Color of the Free Copolymers in the Solid State. In this part, we focus on the impact of the alizarin chemical modification and on its introduction in the macromolecular chains on the resulting polymers. For that, first alizarin and modified alizarin were characterized by UV-visible spectroscopy by diffuse reflectance. Their spectra and respectively their photographs are represented in Figure 3. The comparison of these two spectra shows the shifting of the absorption characteristic band of alizarin from 443 to $428 \mathrm{~nm}$ after modification. These results reach the visual variation of the color of these two compounds: from red-orangey for alizarin to orange-yellowy for modified alizarin. Second, the behavior of incorporated modified dye into polymer chains was also studied. Figure 3 represents photographs and UV-visible diffuse reflectance of two polystyrene with $\mathrm{P}(\mathrm{S}-\mathrm{co}-\mathrm{A})$ and without alizarin (PS), obtained after two hours of polymerization.
Regarding the PS spectrum, which presents absorption band at $256 \mathrm{~nm}$ characteristic of this polymer kind, we notice the appearance on the $\mathrm{P}(\mathrm{S}-\mathrm{co}-\mathrm{A})$ spectrum of absorption bands at 330 and $410 \mathrm{~nm}$. The presence of these two bands signifies the incorporation of modified alizarin into these polymer chains. Also, the yellow color obtained for the $\mathrm{P}(\mathrm{S}-\mathrm{co}-\mathrm{A})$ compared to the white color of PS confirm the UV-visible diffuse reflectance results.

The comparison of spectrum of free and incorporated modified alizarin shows the shifting of dye absorption bands with maximums respectively at 428 and $410 \mathrm{~nm}$ (Figure 3). This result confirms the observed color variation between orange-yellowy and yellow respectively of the free and the incorporated A.

Hybrid Inorganic/Organic Mica-Polymer Pigments. Copolymer Adsorption Density. The adsorption density of polymers on mica platelets can be calculated from the weight lost of the composite which occurs in TGA experiments between 30 and $600{ }^{\circ} \mathrm{C}$ and which corresponds to the degradation of organic phase. The amounts of adsorbed polymer are obtained and represented in Table 1, respectively for composites synthesized after 1, 2, and $4 \mathrm{~h}$.

From the above results, we can notice that the dye content with time in the organic phase is about $1 \%$ by NMR, compared to the styrene units which represents an average value between 0.3 and 2.3 of dyes per macromolecular chain. It means that when the chain grows, the dye content grows simultaneously and with the same incorporation rate of styrene monomers in the macromolecular chains, approximately.

Moreover, it may be noticed that the adsorption density of the polymer chains decreases with the increase of the reaction time. This phenomenon is related to the desorption of the macromolecular chains on a continuous expellation with time, 
Table 1. Organic Composition and Adsorption Density Values for Hybrid Organic/Inorganic Pigments P(S-co-A)/Mica

\begin{tabular}{|c|c|c|c|c|c|c|c|}
\hline \multirow[b]{3}{*}{ composites $\mathrm{P}(\mathrm{S}-\mathrm{co}-\mathrm{A})$} & & & & \multicolumn{4}{|c|}{ adsorption density ( $\left.\sigma_{\text {ads }}\right)$} \\
\hline & \multicolumn{3}{|c|}{ organic composition } & \multicolumn{2}{|c|}{$\mu \mathrm{mol} / \mathrm{mg}$ de mica } & \multicolumn{2}{|c|}{ molecules/nm² } \\
\hline & $M_{\mathrm{n}}(\mathrm{g} / \mathrm{mol})^{a}$ & $\mathrm{~A} / \mathrm{S}$ unit $(\%)^{b}$ & A/PS chain ${ }^{c}$ & PS & $\mathrm{A}^{d}$ & PS & A \\
\hline 1h-mica & 3200 & $\approx 1$ & 0.3 & $4.4 \times 10^{-3}$ & $1.3 \times 10^{-3}$ & 0.448 & 0.135 \\
\hline $2 \mathrm{~h}-$ mica & 8700 & $\approx 1$ & 0.8 & $5.4 \times 10^{-3}$ & $4.5 \times 10^{-3}$ & 0.550 & 0.456 \\
\hline $4 \mathrm{~h}-$ mica & 24000 & $\approx 1$ & 2.3 & $2.9 \times 10^{-3}$ & $6.7 \times 10^{-3}$ & 0.295 & 0.669 \\
\hline
\end{tabular}
per PS chains calculated from $M_{\mathrm{n}} / M_{\text {styrene }} \times\left(\mathrm{A} / \mathrm{S}\right.$ unit). ${ }^{d} \mathrm{~A}=$ alizarin.
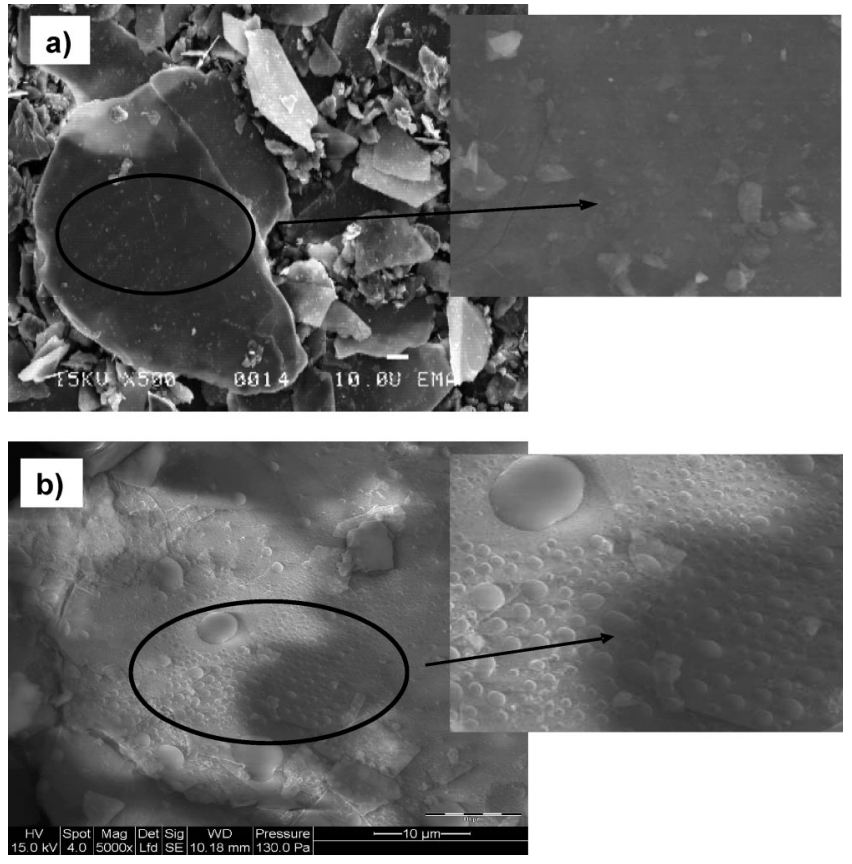

Figure 4. SEM images of (a) pure mica and (b) hybrid organic/inorganic $\mathrm{P}(\mathrm{S}-\mathrm{co}-\mathrm{A}) /$ mica pigment surfaces.

probably due to an increase of the affinity with the bulk. Indeed, when polymer chains grow from the mica surface, the intermolecular interactions with the free polymer chains will be higher than the ionic interactions between the end chains and the oxanions of mica surface. Such phenomenon has already been observed with controlled radical polymerization of butyl acrylate from mica. ${ }^{21}$ Moreover, we can show that the alizarin adsorption density increases due to the increase of the number of alizarin molecules in the chain when chains grow. A robust method has been recently developed by Armes et al. to increase the strong adsorption of macromolecular chains. ${ }^{13}$ They used a cationic polyelectrolyte to enhance the number of interactions by chains and avoid macromolecular desorption.

Copolymer Coating on Mica Surface. The SEM images of pure mica and $\mathrm{P}(\mathrm{S}-\mathrm{co}-\mathrm{A})$ - mica composite surfaces are represented in Figure 4, after washing (see Experimental Section).

The comparison of these two images of pure mica and P(Sco-A)-4h-mica composite shows the appearance of styrene microdroplets on mica surface with practically homogeneous distribution. This submicrometer structure $(\phi<1 \mu \mathrm{m})$ could be associated to a poor wetability at the mica surface due to high interfacial energy mica/PS. Also, the vitreous droplet size (PS $T_{\mathrm{g}}>$ room temperature) is almost identical except in some zones where it seems that several droplets of polymers have coalesced. This droplets morphology of PS on mica surface has been already observed in the past by Suter et al. by initiating the styrene from mica surfaces in presence of peroxides with classical radical polymerization. ${ }^{6-8}$

These SEM images demonstrate that the values of the grafting density expressed in molecule/ $\mathrm{nm}^{2}$ and reported in Table 1 are not representative of the coating state. Indeed, if the grafting density is an experimental value, it is based on a continuous and homogeneous monolayer of PS chains interacting with the mica surface in one end. Here, the SEM images don't really reflect a continuous and homogeneous coating but a inhomogeneous recovery formed by $\mathrm{P}(\mathrm{S}-\mathrm{co}-\mathrm{A})$ microdroplets adsorbed on the inorganic particles surface. Nevertheless, the values expressed in $\mu \mathrm{mol} / \mathrm{mg}$ of mica are really representative of the presence of the organic phase content which is significant in the presence of a multilayer of PS chains on the mica surface. It means that the polymer brushes schematic representation, i.e., chains strongly stretched from the surface, is not appropriate here. Indeed in the microdroplets structured coating, adsorbed polymer chains could be crushed in the mushroom regime surrounded by free PS chains containing the grafted dyes. In this case, some dyes could be closed to the mica/PS interface and the major content far away from it and homogeneously dispersed in the organic coating of droplets. Moreover, the resulting colored hybrid copolymer/mica pigments were repeatedly washed with ethanol, used as a non solvent of the copolymer and a good solvent of the dye. Typically no substantial loss of colorant was observed by UV-visible of the ethanolic phase during the washing step. This is likely due to the grafted colorant in the polymer layer, as described in Figure 2. Subsequent washings do not show a bleeding or leaching of the polymer-coated microsized particles.

Colored Hybrid Copolymer/Mica Pigments. Also, we have observed an interesting effect concerning the variation of colors between free polymer incorporating $\mathrm{A}$ and the same polymer once adsorbed from mica surface. For example, Figure 5 presents the photographs and the absorption UV-vis spectra of the $\mathrm{P}(\mathrm{S}-\mathrm{co}-\mathrm{A})-2 \mathrm{~h}-$ mica composite, the free $\mathrm{P}(\mathrm{S}-\mathrm{co}-\mathrm{A})-2 \mathrm{~h}$ and the PS- $2 \mathrm{~h}-$ mica composite.

On the basis of a "white" mica, the adsorption of a layer of $\mathrm{P}(\mathrm{S}-\mathrm{co}-\mathrm{A})$ on this silicate gave a "dark pink" product, but the color of the free $\mathrm{P}(\mathrm{S}-\mathrm{co}-\mathrm{A})$ (not adsorbed on mica) was yellow. This observation has met previous observation documented in a study of Ghannam et $\mathrm{al}^{21}$ However the PS- $\mathrm{h}-$ mica composite does not show any particular color because it is not incorporating $\mathrm{A}$. The UV-vis diffuse reflectance shows the presence of peak characteristic of observed colors in the corresponding wavelengths: $410 \mathrm{~nm}$ for the yellow free polymer and large band with a maximum at $512 \mathrm{~nm}$ for the pink $\mathrm{P}(\mathrm{S}$ co-A)-2h-mica pigment.

This phenomenon can be attributed to the fact that in the composite, the dye is influenced by the mica surface. Indeed, as previously described, ${ }^{20,21}$ the mica surface has basic character ( $\mathrm{pH} \approx 9$ in water) and as our dye is $\mathrm{pH}$-sensitive (presence of acid phenol group $\mathrm{p} K_{\mathrm{a}} \approx 9$ ), the observed color could correspond to a local effect of the mica surface oxanions on the dye. Indeed, the electrostatic interaction of the alizarin under its acid form $(\mathrm{OH})$ with the mica surface oxanion (basic $\mathrm{SiO}^{-}$or low isoelectric point $\approx 2$ ) can displace and modify, totally or partially, the electronic structure of the alizarin under its basic form $\left(\mathrm{O}^{-}\right)$leading to a shift of its color from yellow to pink (Scheme 2). 


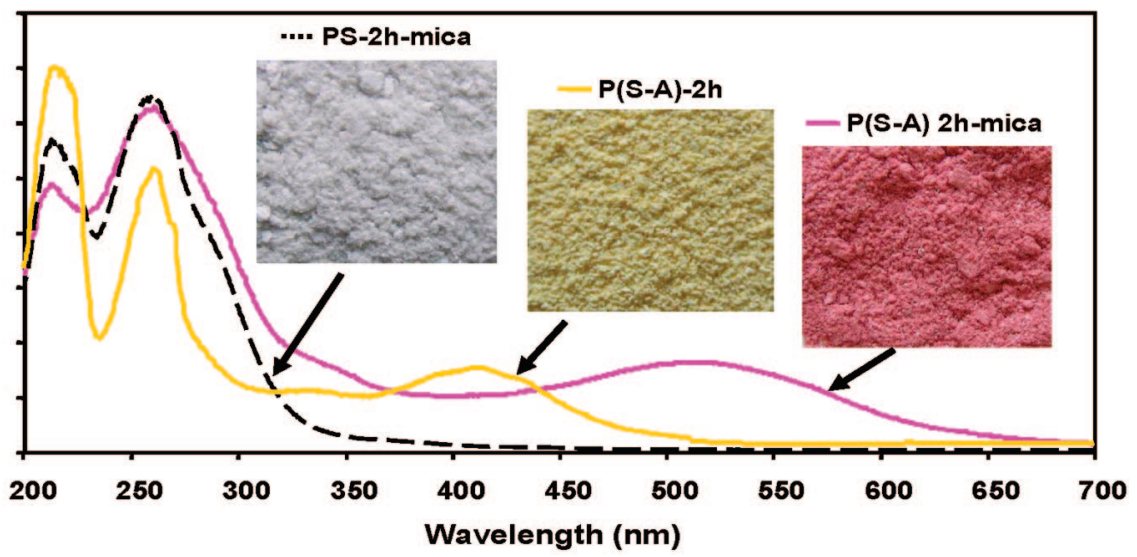

Figure 5. Photographs and UV-vis diffuse reflectance from left to right PS2h/mica, pure $\mathrm{P}(\mathrm{S}-\mathrm{co}-\mathrm{A}) 2 \mathrm{~h}$, and $\mathrm{P}(\mathrm{S}-\mathrm{co}-\mathrm{A}) 2 \mathrm{~h} / \mathrm{mica}$.
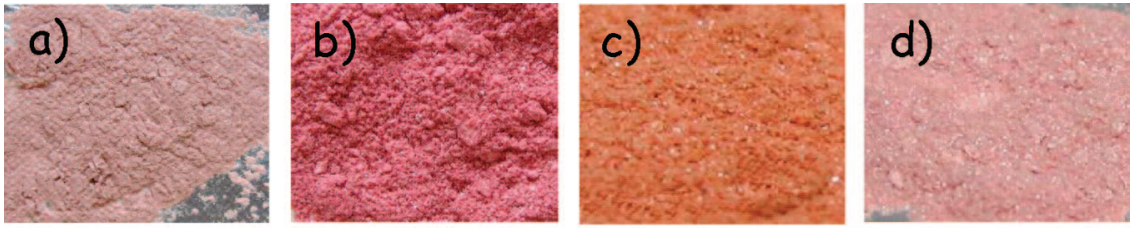

Intensity (a.u)

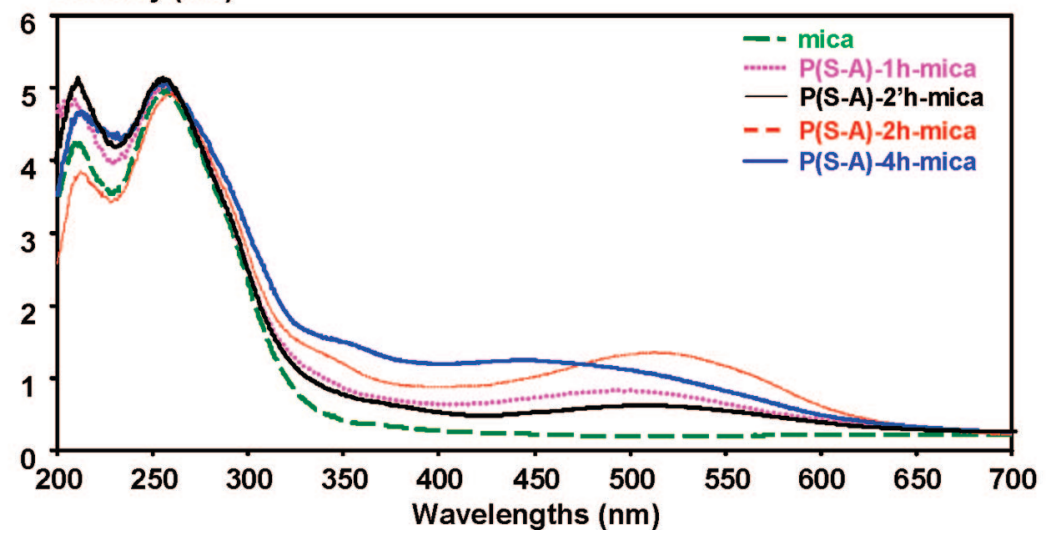

Figure 6. Photographs (bottom) and UV-vis diffuse reflectance (top) from left to right a) $\mathrm{P}(\mathrm{S}-\mathrm{co}-\mathrm{A}) 1 \mathrm{~h} / \mathrm{mica}, \mathrm{b}) \mathrm{P}(\mathrm{S}-\mathrm{co}-\mathrm{A}) 2 \mathrm{~h} / \mathrm{mica}$, c) $\mathrm{P}(\mathrm{S}-\mathrm{co}-$ A) 2 h/mica and d) $\mathrm{P}(\mathrm{S}-\mathrm{co}-\mathrm{A}) 4 \mathrm{~h} / \mathrm{mica}$.

Scheme 3. Photographs of Hybrid Organic/Inorganic Pigments P(S-co-A)/Mica and the Corresponding Schematic Effect of the Dye Interactions with the Mica Surface $($ Dye $=$ Colored Points; Pink = Closed and Yellow = Far Away from the Mica Surface)
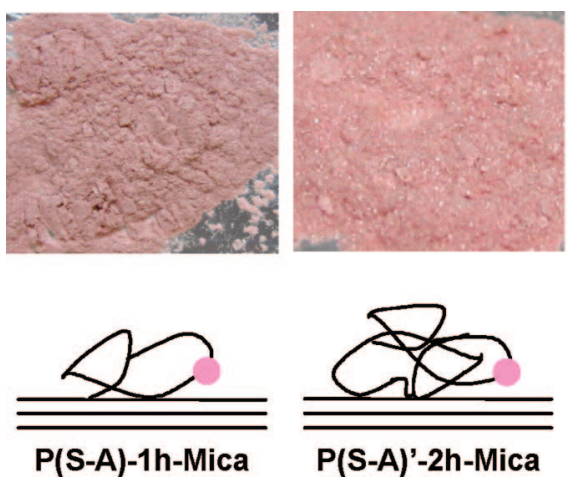

The creation of the negative charge on the alizarin structure is strongly stabilized by its delocalization by mesomer effect around the aromatic structure. In this study, we can not use other characterization methods to support this effect because of the very low content of dye in the organic layer (about 1 mol \%), as previously mentioned (see Table 1). FTIR or ${ }^{13} \mathrm{C}$ NMR solid state can not be used to determine the presence of
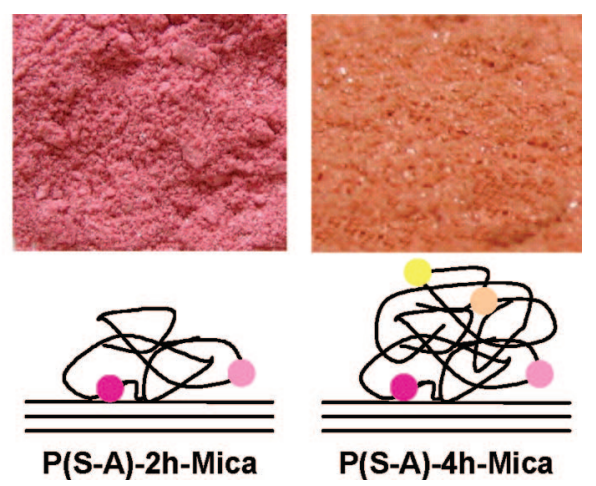

different states or the apparent response of the alizarin in such hybrid inorganic/organic composites. Indeed, the tertiary aromatic carbon sensitive to the electronic delocalization represents only $1 \mathrm{~mol} \%$ of the organic amount and even in ${ }^{1} \mathrm{H}$ NMR in liquid or gel state, it is difficult to be observed (see zoom in Figure 2: hydroxyl peak around $12 \mathrm{ppm}$ ). Moreover, the X-ray photoelectron spectroscopy spectrum of the PS/mica hybrid does 


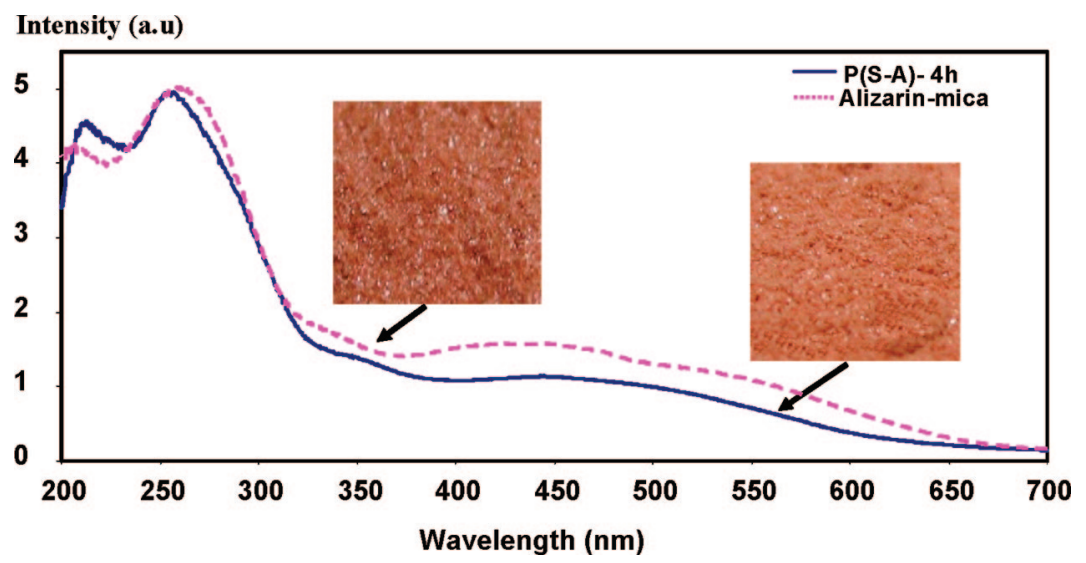

Figure 7. Photographs and UV-vis diffuse reflectance from left to right.
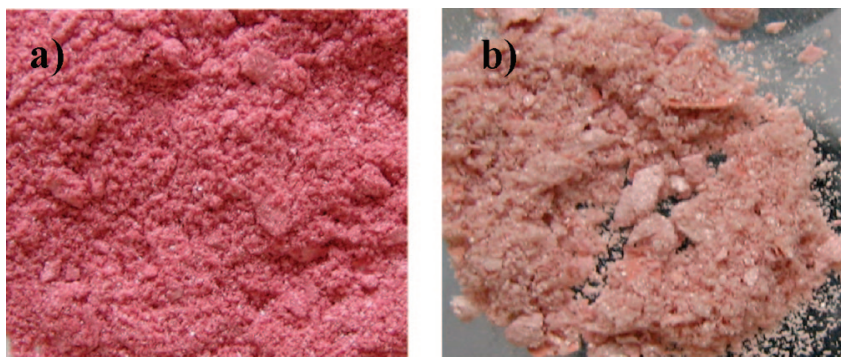

Figure 8. Photographs of $\mathrm{P}(\mathrm{S}-c o-\mathrm{A}) 2 \mathrm{~h} /$ mica (left) and $\mathrm{P}(\mathrm{BA}-b-\mathrm{P}(\mathrm{S}-$ co-A))-mica (right).

not show the presence of aromatic peaks (PS or alizarin) due to residual cation; $\mathrm{K}^{+}(2 \mathrm{p})$ at $293 \mathrm{eV}$ (see Supporting Information). In this case, the $\mathrm{K}^{+}(2 \mathrm{p})$ peak is a shoulder of the main $\mathrm{C}$ (1s) peak at $285 \mathrm{eV}$ and superimposed with the aromatic carbons of the alizarin at high binding energy. Indeed due to the high $\mathrm{K}^{+}$content, the $\mathrm{K}^{+}(2 \mathrm{p})$ peak is superimposed with the $\pi-\pi^{*}$ transition levels $(291 \mathrm{eV})$ of the PS and sensitive alizarin aromatic carbon atoms. In such situation, UV-visible diffuse reflectance is the most appropriated method to support the sensitive effect of the interaction between the grafted dye and the solid substrate, due to the strong adsorption of the dye in this range of wavelength. This characterization has to be correlated with adsorption density by TGA and length of the macromolecular chains by SEC.

It's also interesting to mention that the initiator grafting density of $1.5 \mu \mathrm{mol} / \mathrm{mg}$ of mica corresponds to the complexation of most of the mica surface oxanions, ionically bonded to AIBA. ${ }^{21}$ However, during the polymerization process, we observed a strong desorption of the macromolecular chains associated to a decrease with time of the grafting density values between $4.4 \times 10^{-3}$ and $2.9 \times 10^{-3} \mu \mathrm{mol} / \mathrm{mg}$ of mica (Table 1). This phenomena leads to the enhancement of ionic sites number for electronic interactions of the dye A with the mica particles surface.

Nevertheless, this means that the alizarin color could be used as a local probe of the alizarin position from the surface. Indeed, Figure 6 (top) shows another very interesting result which is based on the color variation of different composites depending on the polymerization time. We found that the color becomes "darker and darker" with the increasing of polymerization time. This can be explained by either the increase of A units incorporated into polymer chains or by the decrease of adsorption density of polymer ( $\sigma_{\text {ads }} \mu \mathrm{mol} / \mathrm{mg}$ of mica) on the mica surface (Table 1).

Notably, for hybrids obtained after 1 and $2 \mathrm{~h}$ of polymerization, we notice from Table 1 that their $\sigma_{\text {ads }}$ are comparable (respectively $4.4 \times 10^{-3}$ and $5.4 \times 10^{-3} \mu \mathrm{mol} / \mathrm{mg}$ of mica).
The color variation observed between these two composites can be then reported to the quantities of A incorporated into polymer chains: more incorporated alizarin, darker observed color.

This hypothesis was verified by preparing $\mathrm{P}(\mathrm{S}-\mathrm{co}$ A) $)^{\prime}-2 \mathrm{~h}-$ mica composite by polymerization of styrene during $2 \mathrm{~h}$ from the mica surface and addition of A after one hour of styrene polymerization. In this case, the $\sigma_{\text {ads }}$ of $\mathrm{P}(\mathrm{S}-\mathrm{co}-$ $\mathrm{A})^{\prime}-2 \mathrm{~h}-$ mica composite is similar to that of $\mathrm{P}(\mathrm{S}-\mathrm{co}-\mathrm{A})-$ $2 \mathrm{~h}-$ mica composite. On the other hand, the color of the $\mathrm{P}(\mathrm{S}$ co-A $)^{\prime}-2 \mathrm{~h}-$ mica composite is comparable to the color of $\mathrm{P}(\mathrm{S}-$ co-A)-1h-mica composite (Figure 6). This hypothesis and these results are in accordance with the calculation of the alizarin quantity into polymer chains, Table 1 , which increases with the polymerization time.

The comparison between UV-vis diffuse reflectance of different composites with the spectrum of mica, Figure 6 (bottom), shows the appearance of characteristic bands of alizarin on the wavelengths corresponding to each color observed for these pigments. However, the characteristic absorption band of styrene at $257 \mathrm{~nm}$ is superimposed to that of $\mathrm{Si}-\mathrm{O}$ of mica.

This comparison leads us to suppose that for equal quantities of mica, only the absorption bands of alizarin change. In this case, the comparison of each spectrum can be done from the intensity of absorption bands of alizarin. However, the UV-vis spectroscopy was not used in any case to quantify the alizarin part in the polymer chains. This is because the high intensity of absorption band of mica at $256 \mathrm{~nm}$ can distort Beer-Lambert law. Despite everything, a simple comparison of spectra can give an idea about the difference of the intensity of alizarin bands.

Figure 6 (bottom) shows the shifting of the maximum of absorption bands of alizarin from 495 to $512 \mathrm{~nm}$ respectively for the $\mathrm{P}(\mathrm{S}-\mathrm{co}-\mathrm{A})$-mica composites obtained after 1 and $2 \mathrm{~h}$ of polymerization. This shifting is accompanied with color variation from clear to dark pink respectively for these two pigments. Also, the difference of absorption intensity of these two bands is a characteristic of the variation of alizarin quantity in adsorbed polymer chains (around two times higher after $2 \mathrm{~h}$ ).

The comparison of $\mathrm{P}(\mathrm{S}-\mathrm{co}-\mathrm{A})^{\prime}-2 \mathrm{~h}-$ mica $\mathrm{UV}-\mathrm{vis}$ diffuse reflectance with those of $\mathrm{P}(\mathrm{S}-\mathrm{co}-\mathrm{A})-1 \mathrm{~h}$ and $2 \mathrm{~h}-$ mica shows that the quantity of polymerized A play an important role in the color of the pigments. In fact, the maximum of absorption is placed, as in the case of $\mathrm{P}(\mathrm{S}-\mathrm{co}-\mathrm{A})-1 \mathrm{~h}-$ mica composite, at 495 $\mathrm{nm}$ with an intensity of the same order. The position and intensity of absorption bands reflect well the color of pigments obtained and especially the effect of A position toward the surface on the color of these pigments.

The observed orange color of $\mathrm{P}(\mathrm{S}-\mathrm{co}-\mathrm{A})-4 \mathrm{~h}-$ mica pigment is confirmed by the presence of a wide absorption band with a 


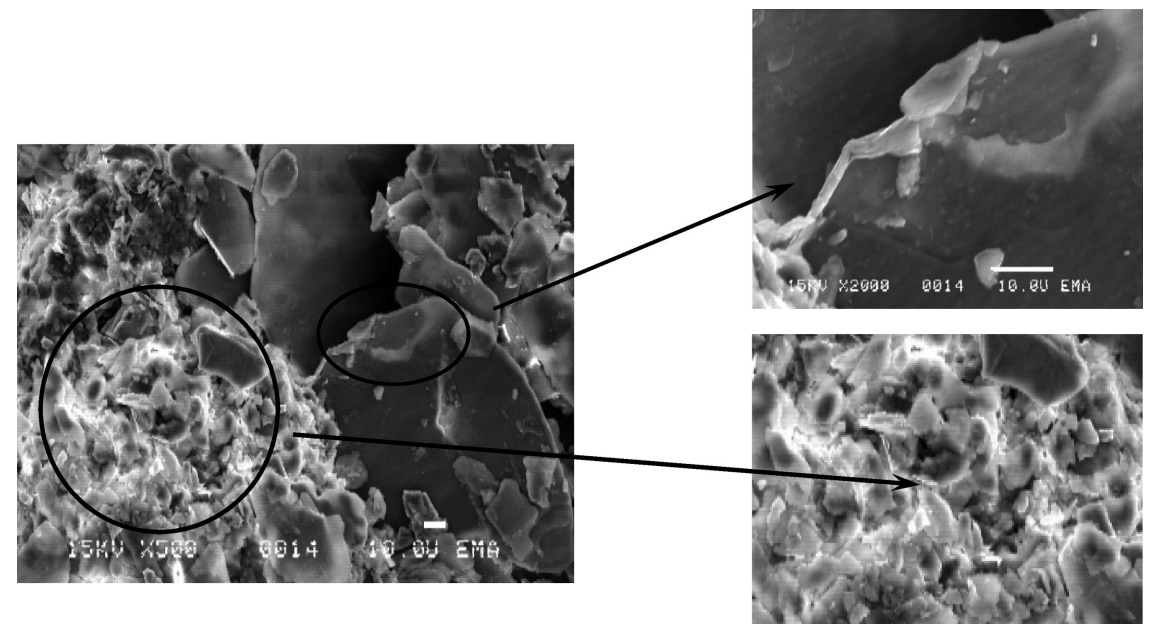

Figure 9. SEM image of hybrid organic/inorganic pigment PBA/mica surface.

maximum at $450 \mathrm{~nm}$ shifting to low wavelengths (from 650 to $390 \mathrm{~nm}$ ). This shift signifies the variation of the alizarin environment.

Concerning the second hypothesis: the variation of pigments color due to the decrease of polymer adsorption density with the increase of polymerization time, the chains are more and more in a "mushroom" regime on the mica surface when adsorption density decreases.

Because our dye is electronic-sensitive, the nearer the chains are to the surface the more the alizarin color will be influenced by the oxanions of mica surface. Consequently, the color should become darker and stretch to purple color, which is not the case of the orange $\mathrm{P}(\mathrm{S}-\mathrm{co}-\mathrm{A})-4 \mathrm{~h}-$ mica pigment color. This could be explained by the fact that not all alizarin molecules interact with the mica surface. The orange color is due to the superimposition of two alizarin colors in the polymer chains: from pink to yellow respectively close to far away from the surface. That corresponds to a "gradient" of alizarin absorption from 510 to $410 \mathrm{~nm}$, respectively (Scheme 3).

This gradient of alizarin composition from the surface to polymer/air interface is also characterized by a broad absorption band, Figure 6 (photo $\mathrm{C}$ ).

To confirm that the aspect of this composite is the result of effects previously described, its color was compared with the color of alizarin-mica compound ( $\sigma_{\text {ads }}$ of $0.8 \mu \mathrm{mol} / \mathrm{mg}$ of mica or 80 molecules $/ \mathrm{nm}^{2}$ ) prepared by simple adsorption of A on the mica surface. This adsorption density is too high to be a simple adsorbed monolayer but represents a superimposition of alizarin molecules at the surface of mica particles as a multilayer of alizarin deposited on mica surface. Figure 7 represents the photographs of $\mathrm{P}(\mathrm{S}-\mathrm{co}-\mathrm{A})-4 \mathrm{~h}-$ mica and alizarinmica as well as their UV-vis diffuse reflections. In this present case, colors are similar which is confirmed by the UV-vis peak positions justifying also our second hypothesis concerning the origin of our pigment colors.

However, a higher color variation can be observed if we compare the $\mathrm{P}(\mathrm{S}-\mathrm{co}-\mathrm{A})-2 \mathrm{~h}-$ mica composite $\left(\sigma_{\text {ads }}\right.$ of 0.55 molecules $\left./ \mathrm{nm}^{2}\right)$ with the $\mathrm{P}(\mathrm{BA}-b-\mathrm{P}(\mathrm{S}-c o-\mathrm{A}))$-mica composite $\left(\sigma_{\text {ads }}\right.$ of 0.07 molecules $/ \mathrm{nm}^{2}$ ) obtained in a previous paper ${ }^{20}$ for the same styrene and modified alizarin polymerization time but from PBA-mica macroinitiator (Figure 8). ${ }^{21}$

We observe that the color of $\mathrm{P}(\mathrm{S}-$ co-A) composite is darker than $\mathrm{P}(\mathrm{BA}-b-\mathrm{P}(\mathrm{S}-c o-\mathrm{A}))$-mica composite. This difference in the color of these two composites confirms our conclusion concerning the effect of adsorption density and, consequently the effect of dye position toward the surface on the composite color. Indeed, for the $\mathrm{P}(\mathrm{BA}-b-\mathrm{P}(\mathrm{S}-c o-\mathrm{A}))-$ mica composite, Figure 8 (right), the mica is enrobed by a PBA monolayer which initiates the second block (S-co-A). The SEM image PBA-mica (macroinitiator) confirms the PBA coating of mica (Figure 9).

In this case, alizarin is partially isolated from the mica surface. This PBA coating restricts the influence of oxanions of mica surface on the dye despite the fact that the composite has a low adsorption density of copolymer which is balanced by the high length of the macromolecular chains. ${ }^{21}$

Indeed, the SEM image of PBA-mica does not show any organization of droplet in the case of PBA-mica. This could be related to the fact that at room temperature the poly(butyl acrylate) is an elastomer polymer having $T_{\mathrm{g}}$ around $-50{ }^{\circ} \mathrm{C}$. Moreover, this polymer should form a homogeneous and regular layer that covers completely the surface of mica particles due to its high affinity with this surface..$^{20,21}$ This homogeneous thin layer can also promote the particles agglomeration as described in Figure 9.

\section{Conclusion}

In summary, we have successfully synthesized varied colored $\mathrm{P}(\mathrm{S}-\mathrm{co}-\mathrm{A})-$ mica pigments by NMP from the surface of mica. Also we have demonstrated that the colors of these synthesized composites depend both on the adsorption density of macromolecular chains on the mica surface and on the dye content without bleeding due to subsequent washing characteristic of a covalent linkage between dye and polymer adsorbed on the micrometer-sized mica particles.

The longer the polymerization time is, the more the chains are crushed onto the mica surface and the nearer the dye is to the surface. In consequence, the dye is more influenced by oxanions of this surface which leads to strong color variations. This variation has been attributed to the superimposition of different electronic states of alizarin: closer to the surface, alizarin is more influenced by oxanions and its color tends to pink, farther away from the surface, the alizarin electronic state is less changed and its color is yellow. Finally, the global color is due to the mix of the different electronic states of alizarin leading to orange pigment. This behavior could be represented as a "gradient" of alizarin concentration from the mica surface to the air/polymer interfaces. It gives us the opportunity to tune the pigment color not only by adjustment of the length and the grafting density but also by the number of dye in chains. These new pigments can be used as fillers in cosmetic or coating applications when they will be dispersed in nitrocellulose or acrylic base, respectively.

Acknowledgment. We would like to thank M. Simon (IPREM ECP) and J. M. Taulmesse (EMA) for UV-visible and SEM 
measurements, respectively. Special thanks go to P. Maury and J. Francois from Gr2PSM for their support and motivation.

Supporting Information Available: Figures showing the dWt/ $d(\ln (M))$ versus $\log (M)$ for unbounded polymers and the XPS spectrum of the $\mathrm{P}(\mathrm{S}-\mathrm{co}-\mathrm{A}) 4 \mathrm{~h} / \mathrm{mica}$ hybrid. This material is available free of charge via the Internet at http://pubs.acs.org.

\section{References and Notes}

(1) Lan, T.; Pinnavaia, T. Chem. Mater. 1994, 6, 2216-2219.

(2) Wang, Z.; Pinnavaia, T. Chem. Mater. 1998, 10, 1820-1826.

(3) Messersmith, P.; Giannelis, E. P. Chem. Mater. 1994, 6, 1719-1725.

(4) Wang, J.; Du, J.; Zhu, J.; Wilkie, C. A. Polym. Degrad. Stab. 2002, $77,249-252$

(5) Zhu, J.; Uhl, F. M.; Morgan, A. B.; Wilkie, C. A. Chem. Mater. 2001, $13,4649-4654$

(6) Meier, L. P.; Shelden, R. A.; Caseri, W. R.; Suter, U. W. Macromolecules 1994, 27, 1637-1642.

(7) Velten, U.; Tossati, S.; Shelden, R. A.; Caseri, W. R.; Suter, U. W. Langmuir 1999, 15, 6940-6945.

(8) Velten, U.; Shelden, R. A.; Caseri, W. R.; Suter, U. W.; Li, Y. Colloids Surf. A: Physicochem. Eng. Asp. 1999, 154 (1), 87-96.

(9) Von Werne, T.; Patten, T. E. J. Am. Chem. Soc. 1999, 121, 74097410

(10) Von Werne, T.; Patten, T. E. ACS PMSE Prep. 2000, 82 (1), 294.
(11) Matsuno, R.; Yamamoto, K.; Ostuka, H.; Takahara, A. Chem. Matter. 2003, 15, 3-5.

(12) Matsuno, R.; Otsuka, H.; Takahara, A. Soft Matter 2006, 5, 415-421.

(13) Chen, M.; Briscoe, W. H.; Armes, S. P.; Cohen, H.; Klein, J. ChemPhysChem. 2007, 8, 1303-1306.

(14) Matyjaszewski, K., Ed. Advances in Controlled/Lining Radical Polymerization; ACS Symposium Series 854; American Chemical Society: Washington, DC, 2003.

(15) Bartholome, C.; Beyou, E.; Bourgeat-Lami, E.; Chaumont, P.; Zydowicz, N. Macromolecules 2004, 38, 1099-1106.

(16) (a) Parvole, J.; Montfort, J. P.; Billon, L. Macromol. Chem. Phys. 2004, 205, 1369-1378. (b) Parvole, J.; Laruelle, G.; Khoukh, A.; Billon, L. Macromol. Chem. Phys. 2005, 206, 372-382.

(17) Inoubli, R.; Dagreou, S.; Roby, F.; Khoukh, A.; Peyrelasse, J.; Billon, L. Polymer 2005, 46, 2486-2496.

(18) Inoubli, R.; Dagreou, S.; Lapp, A.; Billon, L.; Peyrelasse, J. Langmuir 2006, 22, 6683-6689.

(19) Inoubli, R.; Dagreou, S.; Delville, M. H.; Lapp, A.; Peyrelasse, J.; Billon, L. Soft Matter 2007, 3, 1014-1024.

(20) Ghannam, L.; Garay, H.; Shanahan, M. E. R.; Francois, J.; Billon, L. Chem. Mater. 2005, 17, 3837-3843.

(21) Ghannam, L.; Bacou, M.; Garay, H.; Francois, J.; Shanahan, M. E. R.; Billon, L. Polymer 2004, 45, 7035-7045.

(22) Ghannam, L.; Garay, H.; Billon, L. Macromol. Chem. Phys. 2007, 208, 1469-1479.

MA800522K 